The American

Society of Pediatric

Neurosurgeons

\section{8th Annual Meeting of the American Society of Pediatric Neurosurgeons}

January 25-30, 2015 • Fairmont Orchid, Kona, Hawaii

Program
Monday, January 26

\author{
6:45 AM-7:20 AM \\ Breakfast for Registered \\ Physicians
}

7:20 AM-7:30 AM

Opening Remarks

Alan R. Cohen, MD, ASPN President;

Gerald Grant, MD, ASPN Scientific

Program Chair

7:30 AM-9:15 AM

General Session I: TUMOR I

Moderators: John A. Duncan III, MD,

$\mathrm{PhD}$, Santa Clara, CA; Jeffrey H. Wisoff,

MD, New York, NY

7:30 AM-7:45 AM

Complication Case \#1

Alan R. Cohen, MD, Boston, MA

7:45 AM-8:00 AM

Complication Case \#2

Eric Jackson, MD, Columbus, $\mathrm{OH}$

8:00 AM-8:15 AM

Complication Case \#3

Sean McNatt, MD, Roseville, CA

8:15 AM-8:30 AM

Complication Case \#4

Paul Steinbok, MB, BSc, Vancouver, BC

8:30 AM-8:45 AM

Complication Case \#5

Peter B. Dirks, MD, PhD, Toronto, ON

8:45 AM-9:00 AM

Complication Case \#6

David I. Sandberg, MD, Houston, TX

9:00 AM-9:15 AM

Complication Case \#7

John C. Wellons, MD, MSPH, Nashville, TN

9:15 AM-9:30 AM

Complication Case \#8

Lance S. Governale, MD, Columbus, $\mathrm{OH}$

9:30 AM-9:45 AM

Beverage Break
9:45 AM-10:45 AM

General Session II: TRAUMA

Moderators: P. David Adelson, MD,

Phoenix, AZ; Mark S. Dias, MD, Hershey, PA

9:45 AM-10:00 AM

Complication Case \#1

Mark S. Dias, MD, Hershey, PA

10:00 AM-10:15 AM

Complication Case \#2

David J. Donahue, MD, Ft. Worth, TX

10:15 AM-10:30 AM

Minimizing Radiation Exposure

in Evaluation of Pediatric Head

Trauma: Utilization of Rapid MR

Imaging

Avinash Mohan, MD, Valhalla, NY

10:30 AM-10:45 AM

Complications Following

Pediatric Cranioplasty after

Decompressive Craniectomy: a

Multicenter Retrospective Study

Brandon Rocque, MD, MS, Birmingham, $\mathrm{AL}$

10:45 AM-11:00 AM

Beverage Break

11:00 AM-12:30 PM

General Session III:

ENDOSCOPY

Moderators: James M. Drake, MD,

Toronto, ON; Mark M. Souweidane, MD,

New York, NY

11:00 AM-11:15 AM

Complication Case \#1

Nathan R. Selden, MD, PhD, Portland, OR

11:15 AM-11:30 AM

Complication Case \#2

James M. Drake, MD, Toronto, ON

11:30 AM-11:45 AM

Complication Case \#3

Sean Lew, MD, Milwaukee, WI

11:45 AM-12:00 PM

Complication Case \#4

Mark M. Souweidane, MD, New York, NY
12:00 PM-12:15 PM

Complication Case \#5

Edward Ahn, MD, Baltimore, MD

12:15 PM-12:30 PM

Complication Case \#6

Michael L. Levy, MD, PhD, San Diego, CA

Tuesday January 27

6:45 AM-7:30 AM

Breakfast for Registered

Physicians

7:30 AM-9:00 AM

General Session IV: VASCULAR

Moderators: Michael S. B. Edwards, MD,

Palo Alto, CA; Cormac O. Maher, MD,

Ann Arbor, Ml

7:30 AM-7:45 AM

Complication Case \#1

Mark D. Krieger, MD, Los Angeles, CA

7:45 AM-8:00 AM

Complication Case \#2

Lenwood P. Smith Jr., MD, Greenville, NC

8:00 AM-8:15 AM

Complication Case \#3

John S. Myseros, MD, Washington, DC

8:15 AM-8:30 AM

Complication Case \#4

James T. Rutka, MD, Toronto, ON

8:30 AM-8:45 AM

Complication Case \#5

Dale M. Swift, MD, Dallas, TX

8:45 AM-9:00 AM

Complication Case \#6

Lissa C. Baird, MD, Portland, OR

9:00 AM-9:15 AM

Beverage Break

9:15 AM-10:15 AM

HAROLD AND JOANN HOFFMAN

LECTURESHIP

Why Most Published Research

Findings Are False

John P. A. loannidis, MD, Stanford

University, Palo Alto, CA 
10:15 AM-10:30 AM

Beverage Break

10:30 AM-11:45 AM

General Session V:

\section{HYDROCEPHALUS I}

Moderators: Ann Marie Flannery, MD, St.

Louis, MO; Herbert E. Fuchs, MD, PhD,

Durham, NC

10:30 AM-10:45 AM

Complication Case \#1

Philipp R. Aldana, MD, Jacksonville, FL

10:45 AM-11:00 AM

Complication Case \#2

Raphael Guzman, MD, Basel,

Switzerland

11:00 AM-11:15 AM

Complication Case \#3

Andrew Parent, MD, Jackson, MS

11:15 AM-11:30 AM

Complication Case \#4

Scellig Stone, MD, PhD, Boston, MA

11:30 AM-11:45 AM

Use of Silver-Impregnated

Dressings to Reduce

Neurosurgical Infections

Prithvi Narayan, MD, Philadelphia, PA

11:45 AM-12:15 PM

Symposium: Washington Update

11:45 AM-12:00 PM

The Elections are Over: Now

What?

Katie O. Orrico, JD, Director, AANS/CNS

Washington Office, Washington, DC

12:00 PM-12:15 PM

The Perils of Politics

Monica C. Wehby, MD, Portland, OR

\section{Wednesday, January 28}

6:45 AM-7:30 AM

Breakfast for Registered

Physicians

6:45 AM-7:30 AM

Breakfast for Fellowship Program

Directors

7:30 AM-9:30 AM

General Session VI: TUMOR II

Moderators: John A. Duncan III, MD,

PhD, Santa Clara, CA; Jeffrey H. Wisoff,

MD, New York, NY

\section{7:30 AM-7:45 AM}

Complication Case \#1

John Ragheb, MD, Miami, FL
7:45 AM-8:00 AM

Complication Case \#2

Jodi L. Smith, Indianapolis, IN

8:00 AM-8:15 AM

Complication Case \#3

Matthew D. Smyth, MD, St. Louis, MO

8:15 AM-8:30 AM

Complication Case \#4

Michael H. Handler, MD, Aurora, CO

8:30 AM-8:45 AM

Complication Case \#5

P. David Adelson, MD, Phoenix, AZ

8:45 AM-9:00 AM

Complication Case \#6

Zulma Tovar-Spinoza, MD, Syracuse, NY

9:00 AM-9:15 AM

Complication Case \#7

Clarence S. Greene Jr., MD, New

Orleans, LA

9:15 AM-9:30 AM

Complication Case \#8

Tadanori Tomita, MD, Chicago, IL

9:30 AM-9:45 AM

Beverage Break

9:45 AM-10:00 AM

Targeting Histone Mutations in

Pediatric Glioblastomas

David J. Daniels, MD, PhD, Rochester, MN

10:00 AM-10:15 AM

Convection-Enhanced Drug

Delivery to Pediatric Brain

Tumors-Individual Tumor

Physiology and not Target

Specificity will Determine

Efficacy

Peter C. Warnke, MD, Chicago, IL

10:15 AM-11:00 AM

General Session VII: SPINE I

Moderators: Douglas L. Brockmeyer, MD, Salt Lake City, UT; Andrew Jea, MD, Houston, TX

10:15 AM-10:30 AM

Complication Case \#1

Andrew Foy, MD, Milwaukee, WI

10:30 AM-10:45 AM

Complication Case \#2

Andrew Jea, MD, Houston, TX

10:45 AM-11:00 AM

Complication Case \#3

Todd C. Hankinson, MD, MBA, Aurora, $\mathrm{CO}$

11:00 AM-11:15 AM

Beverage Break
11:15 AM-12:15 PM

General Session VIII: SPINE II

Moderators: Douglas L. Brockmeyer,

MD, Salt Lake City, UT; Andrew Jea, MD,

Houston, TX

11:15 AM-11:30 AM

Complication Case \#1

Phillip B. Storm, MD, Philadelphia, PA

11:30 AM-11:45 AM

Complication Case \#2

Mahmoud Nagib, MD, Minneapolis, MN

11:45 AM-12:00 PM

Using iMR and iCT Together

in Severe Pediatric Deformity

Correction: Cervicothoracic

Kyphosis with Myelopathy in

a 13 Year Old Female with

Chondrodysplasia Punctata

David F. Bauer, MD, Lebanon, NH

12:00 PM-12:15 PM

Use of C1 Lateral Mass Screws

in the Reduction of Atlanto-Axial

Deformity: Technical Note

Jonathan E. Martin, MD, Hartford, CT

\section{Thursday, January 29}

6:45 AM-7:30 AM

Breakfast for Registered

Physicians

7:30 AM-9:30 AM

General Session IX: CHIARI

Moderators: Richard G. Ellenbogen,

MD, Seattle, WA; W. Jerry Oaks, MD,

Birmingham, AL

7:30 AM-7:45 AM

Complication Case \#1

Michael J. Burke, MD, Corpus Christi, TX

7:45 AM-8:00 AM

Complication Case \#2

David M. Frim, MD, Chicago, IL

8:00 AM-8:15 AM

Complication Case \#3

David F. Jimenez, MD, San Antonio, TX

8:15 AM-8:30 AM

Complication Case \#4

Curtis J. Rozzelle, MD, Birmingham, AL

8:30 AM-8:45 AM

Complication Case \#5

R. Michael Scott, MD, Boston, MA 
8:45 AM-9:00 AM

Complication Case \#6

Mark M. Souweidane, MD, New York, NY

9:00 AM-9:15 AM

Complication Case \#7

Andrew Jea, MD, Houston, TX

9:15 AM-9:30 AM

Complication Case \#8

Nicholas M. Wetjen, MD, Rochester, MN

9:30 AM-9:45 AM

Beverage Break

9:45 AM-10:15 AM

General Session X: EPILEPSY I

Moderators: Jeffrey G. Ojemann, MD,

Seattle, WA; James T. Rutka, MD,

Toronto, ON

9:45 AM-10:00 AM

Complication Case \#1

Philipp R. Aldana, MD, Jacksonville, FL

10:00 AM-10:15 AM

Complication Case \#2

Daniel Curry, MD, Houston, TX

10:15 AM-10:45 AM

INTERNATIONAL INVITED LECTURER

Operating in the Cradle:

Neurosurgery in Africa

Graham Fieggen, PhD, Cape Town,

South Africa

10:45 AM-11:00 AM

Beverage Break

11:00 AM-11:45 AM

General Session XI: EPILEPSY II

Moderators: Jeffrey G. Ojemann, MD,

Seattle, WA; James T. Rutka, MD,

Toronto, ON

11:00 AM-11:15 AM

Complication Case \#1

James Baumgartner, MD, Orlando, FL

11:15 AM-11:30 AM

Complication Case \#2

Sandeep Sood, DO, Detroit, MI

11:30 AM-11:45 AM

Complication Case \#3

Howard L. Weiner, MD, New York, NY

11:45 AM-12:15 PM

Business Meeting-Open to ASPN

Members Only
12:30 PM-6:00 PM

ASPN Golf and Tennis

Tournaments

6:30 PM-10:00 PM

Gala Banquet

Friday, January 30

\author{
6:45 AM-7:30 AM \\ Breakfast for Registered \\ Physicians
}

7:30 AM-8:45 AM

General Session XII:

HYDROCEPHALUS II

Moderators: Ann Marie Flannery, MD, St. Louis, MO; Herbert E. Fuchs, MD, PhD,

Durham, NC

7:30 AM-7:45 AM

Complication Case \#1

David D. Cochrane, MD, Vancouver, BC

7:45 AM-8:00 AM

Complication Case \#2

Ann Marie Flannery, MD, St. Louis, MO

8:00 AM-8:15 AM

Endoscopic Third

Ventriculostomy and

Ventriculoperitoneal Shunting: A

Comparative Effectiveness Study

Using Administrative Claims

Data

Sandi Lam, MD, Houston, TX

8:15 AM-8:30 AM

Efficacy of Hydromer-Coated

Shunt Systems in Reducing Early

Shunt Infections

Ivan J. Sosa, MD, San Pablo, PR

8:30 AM-8:45 AM

Ciliary Contributions to

Progenitor Differentiation in

Hydrocephalus

Timothy W. Vogel, MD, Cincinnati, $\mathrm{OH}$

8:45 AM-9:00 AM

Beverage Break

9:00 AM-10:15 AM

General Session XIII:

CRANIOFACIAL

Moderators: Robert F. Keating, MD,

Washington, DC; Steven J. Schiff, MD,

$\mathrm{PhD}$, University Park, PA
9:00 AM-9:15 AM

Complication Case \#1

Graham Fieggen, PhD, Cape Town,

South Africa

9:15 AM-9:30 AM

Complication Case \#2

Stephen J. Haines, MD, Minneapolis, MN

9:30 AM-9:45 AM

Complication Case \#3

Mark Proctor, MD, Boston, MA

9:45 AM-10:00 AM

Complication Case \#4

Michael H. Handler, MD, Aurora, CO

10:00 AM-10:15 AM

Complication Case \#5

Daniel J. Guillaume, MD, Minneapolis, MN

10:15 AM-10:30 AM

Beverage Break

10:30 AM-11:00 AM

General Session XIV:

SPASTICITY

Moderators: Michael D. Partington, MD,

St. Paul, MN; Shenandoah Robinson,

MD, Boston, MA

10:30 AM-10:45 AM

Complication Case \#1

Michael D. Partington, MD, St. Paul, MN

10:45 AM-11:00 AM

Complication Case \#2

Shenandoah Robinson, MD, Boston, MA

11:00 AM-11:30 AM

General Session XV: SPINA

BIFIDA

Moderators: Karin M. Muraszko, MD, Ann Arbor, MI; Benjamin C. Warf, MD, Boston, MA

11:00 AM-11:15 AM

Complication Case \#1

Elizabeth Tyler-Kabara, MD, PhD,

Pittsburg, PA

11:15 AM-11:30 AM

Complication Case \#2

Gerald F. Tuite, MD, St. Petersburg, FL

11:30 AM

Meeting Adjourns 